\title{
LEADING AND WORKING IN TEAMS
}

Professor Helen O'Sullivan, Professor of Medical Education, School of Medicine, University of Liverpool

Dr Michael J Moneypenny, Director of Scottish Clinical Simulation Centre, Forth Valley Royal Hospital, Larbert

Professor Judy McKimm, Director of Strategic Educational Development, College of Medicine, Swansea University

\section{CONFLICT OF INTERESTS}

The authors have no conflicts of interest to declare in the writing of this article.

This article considers the role of the clinical leader as a team member and leader and explores how an understanding of the purpose and functions of teams can help doctors work more effectively in the various teams with which they are involved.

\section{INTRODUCTION}

Healthcare is primarily delivered by a range of health workers and managers working in a number of interlinked teams. Effective teamwork is increasingly important due to the complexity and specialisation of care; ageing populations with co-morbidities and rise of long term-conditions; global workforce shortages; changes in skills mix of health workers; safe working hours' initiatives and shifts towards more integrated health and public services. 'Our challenge is not whether we will deliver in teams, but rather how well we deliver in teams' (Schyve, 2005). Given the importance of teams to the delivery of effective health care, clinical leaders need to be able to lead, work within and between teams as seamlessly as possible. Understanding what makes teams function well and less effectively can help leaders overcome some of these teamworking challenges.

The teamSTEPPS program identifies different, inter-related team types that support and deliver healthcare:

1. Core teams - involved in direct patient care, usually (but not always) based where the patient receives care.

2. Co-ordinating teams - responsible for operational and resource management and allocation. 
3. Contingency teams - emergent, crisis, time-limited, formed from various core team members.

4. Ancillary and support services - service delivery e.g. cleaners, porters, catering, medical records.

5. Administration - executive leadership, define culture, policies, staff expectations (Quality AfHRa, 2007).

\section{DRIVERS FOR IMPROVED TEAM WORKING AND LEADERSHIP IN HEALTHCARE}

Whether as components of clinical competence and communication skills, commitment to professional competence, or working in partnership, effective leadership and teamwork are increasingly recognised as essential skills in clinical care (e.g. Francis, 2013). In the UK, the National Confidential Enquiry into Maternal Deaths stated that poor teamwork was a leading cause of substandard obstetric care (Cooper and McClure, 2005). In the US, the Institute of Medicine's report "Crossing the Quality Chasm" emphasised the need for improved leadership and teamwork in clinical practice (Chakraborti et al., 2008). Hjortdahl et al. (2009) suggested that effective leadership improves team performance and goal achievement and other research has shown that good teamwork reduces errors, reduces mortality and morbidity rates and improves patient safety (Neily et al., 2010) From a social perspective, as the population ages, more patients will present with multiple health problems, requiring effective interdisciplinary teamwork and leadership (Xyrichis et al, 2008).

Since 2009, a number of high-profile inquiries into poor healthcare have made clear links between leadership, multidisciplinary teamwork, high quality healthcare and good health outcomes. The Francis (2013) report detailed the failings in care at the Mid-Staffordshire NHS trust. Poor leadership, by nursing, medical and boardroom staff was highlighted as a particular area of concern. It also called for "effective teamwork between all the different disciplines and services" (p.110). The report also emphasised the importance of good leadership: "The common culture and values of the NHS must be applied at all levels of the organization, but of particular importance is the example set by leaders" (p.78). The Keogh Mortality Review (Keogh, 2013) subsequently reported on 14 hospitals with high standardised mortality ratios. Poor leadership was again identified as a cause of patient harm. The Prime Minister then asked Don Berwick, former president of the US Institute for Healthcare Improvement, to report on patient safety. His report - "A promise to learn - a commitment to act: Improving the Safety of Patients in England" (National Advisory Group on the Safety of Patients in England, 2013) - recommended that "All NHS leaders and managers should actively address poor teamwork" (p.16) and gave guidance on the shift in leadership behaviours required. 
Ezziane et al (2012) suggested that the key areas for consideration when leading or building a healthcare team are communication, decision-making, patient safety, conflict resolution and identifying appropriate roles for individual team members.

Communication - as noted above, adverse events resulting from error happen at unacceptably high rates in the inpatient setting, with ineffective or insufficient communication among team members being a contributing factor. Communication through means such as e-mail, has increased in the last ten years, removing several important aspects of interaction, potentially fragmenting and isolating health care workers, rather than encouraging team-building. Clinical leaders must therefore look for appropriate methods of communication to better direct their teams. Regular meetings that create an environment that welcomes independent expression of a team member's views are particularly important (Ezziane et al 2012).

Tools that help team members communicate include SBAR (Situation, Background, Assessment, Recommendation); Callout; Check-back and Handover or Handoff (e.g. 'I pass the baton' - Introduction, Patient, Assessment, Situation, Safety concerns, Background, Actions, Timing, Ownership, Next) (World Health Organisation, 2011).

Decision making - In a group or team setting, a leader has to be aware of the tendency for "group think" where members of the team go along with decisions for fear of being ostracised for challenging a decision. This is linked to the issues of leadership and hierarchy discussed in the section on "power distance" below. Leaders of a healthcare team need to find a leadership style that encourages challenge and nurtures independent thought.

Patient Safety - Patient safety is a key focus on improving healthcare in the UK (Institute for Healthcare Improvement, 2015). Healthcare teams have learned from other sectors such as the aviation industry in procedural and mechanised ways of cutting down on errors. Setting clear goals around patient safety and quality improvement help to focus team members' activities towards a patient-centred approach to care.

Conflict Resolution - One of the downsides of precluding "groupthink" from a team is a potential increase in conflict. Following disagreement there is potential for confrontation and escalation to occur and can create long-term disharmony in teams. It is therefore important that a clinical leader is able to foster negotiation and compromise in such situations, more specifically aiming towards group-trust, shared commitments and mutual respect of opposing views (Ezziane et al 2012).

The WHO Patient safety Guide (2011) describes three useful tools to help empower team members: the 'two-challenge rule' (voicing and restating concerns at least twice); CUS (I am Concerned, I am Uncomfortable, this is a Safety issue), a three-step process for assisting people in stopping an activity; DESC Script (Describe the specific situation/behaviour/issue; 
Express how the situation makes you feel; Suggest other alternatives; state the Consequence) for resolving conflict.

Identifying appropriate roles - Porter-O'Grady et al. (2010) suggests that that failure of role-assignment to team members is one of the most significant causes of stress in the work place. Several methods and types of analysis can be employed to ensure that there is clarity of role and purpose in the team.

The above points are reinforced by West et al. (2015) in their review of the evidence base for leadership in healthcare. Specifically referring to team working and leadership, they note that:

- Effective team working is essential for organisational success;

- 'Leadership clarity is associated with clear team objectives, high levels of participation, commitment to excellence and support for innovation'(p12);

- Conflict within teams leads to poor outcomes and processes;

- Shared leadership is a predictor of team effectiveness.

Specifically we should ask: What are we trying to accomplish? How will we know that a change is an improvement? What changes can we make that will result in improvement? (Institute for Healthcare Improvement, 2015).

West and Lyubovnikova (2013) suggest that teams which have low levels of interdependence, shared objectives and reflectivity are known as 'pseudo-teams' - whilst they may appear team-like, they have few of the characteristics of effective, high performing teams. The detrimental impact of such pseudo-teams is compounded in that most health professionals work in many teams in different contexts and over time (O'Leary et al., 2011). Being able to work in multiple teams therefore requires an adaptive mix of flexibility, credibility and authenticity, and leaders need also to be able to effect communication and manage activities between teams.

\section{MULTI-PROFESSIONAL TEAMS}

As services become more integrated and person-centred, health workers are increasingly working in multi professional teams. Multi-professional teamworking can be defined as:

"A dynamic process involving two or more health professionals with complementary backgrounds and skills, sharing common health goals and exercising concerted physical and mental effort in assessing, planning, or evaluating patient care. This is accomplished through interdependent collaboration, open communication and shared decision-making. This in turn generates value-added patient, organisational and staff outcomes." (Xyrichis, 2009, p238)

Whatever the makeup of the team, research has shown that the quality of leadership is crucial to improved outcomes to patients and that an engaging, authentic and shared leadership approach is the most effective (West et al., 2015). Whilst leading or working 
within uni-professional teams can be very challenging, additional and specific challenges exist when working in multi-professional teams.

Firstly, multi-professional teams tend to have complex structures. Whilst uni-professional teams usually have a single reporting line, multi professional teams often have more complex structures - perhaps reporting to different senior managers, and having separate supervisory and, often, funding arrangements. Secondly, multi-professional teams include a range of different professionals. The leader typically comes from a background in one profession and they will have to gain the respect of the full range of professions within the team. Acknowledging and working with potential issues of authority, power and control and resolving conflicts are essential skills for team leaders (Barrow et al., 2104). Leaders who can work adaptively within such complexity are likely to be more successful.

It is vital that leaders establish and maintain credibility but this can be difficult when they are responsible for other professionals, clinical practice or activities that did not form part of their education or training. Working with, motivating, leading and supervising people with a range of values and skills bases raises issues about "professional identity" (Anning et al., 2010). Professional identity (the values and scope of practice that defines a profession) can be very positive, it binds people together and helps them feel that they belong to a community of practice. However, because each professional identity requires people to see members of professions different from theirs as 'the other', this can lead to 'in groups', 'out groups', misunderstandings and miscommunications. Leaders who understand this and can negotiate and agree common values, goals and approaches to care will help bring team members together around a shared purpose and way of working, despite their professional backgrounds.

Leaders of multi-professional teams also need to be "boundary crossers" (Mathur and Skelcher, 2007), that is, have the ability to work with a range of professional groups in a way that engenders confidence. They will need to take a 'translational' role, learn to speak the 'language' of different professional groups (including health managers) and demonstrate respect for all team members' perspectives. A distributed or shared leadership style will be a good fit in most contexts and the leader will need to strike a balance between maintaining an authoritative and confidence-inspiring leadership style and being able to admit when they don't have sufficient knowledge about a profession to make a decision.

\section{TEAM WORKING AND POWER RELATIONS}

Barriers to effective teamworking include changing roles; changing settings; medical hierarchies; individualistic nature of medicine and instability of teams (WHO, 2011). In medicine, physicians have traditionally been at the top of the power structure and, consequently, have the greatest potential to impact those around them, including patients and other members of the healthcare team. This phenomenon is commonly referred to as 
"power distance." Power distance occurs when individuals in positions of less power are reluctant to challenge those with greater authority and can lead to detrimental outcomes for patients and unhelpful ways of working such as sabotage, working around and passive aggression or rebellion (Barrow et al., 2014). In some environments, such as the battlefield or emergency situations, strict adherence to the established power structures is vital but in other contexts, power distance may actually result in harm. The power distance index was part of Hofstede's cultural dimensions theory which given insight into the impact of cultural difference and leadership in global business (Hofstede, 1991). Using a low power distance management or negotiation approach (i.e. engaging or nearby leadership) with someone accustomed to a high power distance culture may be counter-productive and vice versa. Power distance may also describe the leadership relationship between doctors and other healthcare professionals, especially nurses.

Whilst leader-follower relationships involve dominance and some deference, this can be catastrophic in consequence when people feel they cannot speak out. The airline industry has many examples of where the hierarchical nature of the leadership amongst the crew resulted in catastrophe for example the crash of Korean Air Flight 801 in 1997 was attributed primarily to the rigidly hierarchical power structure in the cockpit, which prevented crew from speaking up until it was too late. In medicine, lack of clarity in the leadership structure and inability to challenge the leadership decisions of others can be equally catastrophic. In March 2005, Elaine Bromiley, a 37-year-old mother of two, was scheduled to undergo a routine sinus operation under general anaesthesia. Unfortunately there were complications with managing her airway after she had been anaesthetised. Two consultant anaesthetists and a consultant ENT surgeon were unable to obtain a definitive airway and she suffered hypoxic brain damage. Her life support was switched off some days later. An Independent Report into her death criticised the lack of communication within the team (Harmer, 2007). Her husband, Martin Bromiley, an airline pilot and expert in human factors training in aviation, stated:

"The lead anaesthetist... in his own words 'lost control'. There was a question mark, in the inquest, about who people felt was in charge at different points... There was certainly a breakdown in the decision-making processes and it would appear that the communication processes dried up amongst the consultants." (Clinical Human Factors Group, 2008)

In a recent study, medical students were placed in a simulated acute situation as part of their course on leadership and professionalism. As sample of the students were placed in situation where a senior colleague made a deliberate and potentially life threatening error. Where students didn't challenge the senior colleague, the most common reason for not speaking up was "assumed hierarchy", i.e. the senior is not questioned simply because they are more senior, rather than perceived to be more experienced (the second most common reason for not speaking up (Moneypenny et al 2013). In a similar simulator-based study, St 
Pierre et al. (2012) looked at the willingness of residents and nursing staff to challenge deliberate errors committed by attending physicians. They found that the attending was only challenged in $28 \%$ of situations. When they did challenge they used crisp advocacyinquiry $(40 \%)$, an oblique statement $(35 \%)$ or addressed the problem without pursuing it further (25\%). When asked why they did not challenge, $37 \%$ had no answer, $35 \%$ admitted to there being a discrepancy between what they knew and what they did, $12 \%$ explained that the authority gradient prevented them from speaking up, while $8 \%$ stated that attendings routinely violated standard operating procedures (SOPs) without being challenged.

Responses to adverse events and reports on teamworking routinely emphasise the need to move away from a hierarchical, 'command and control' leadership style to one of distributed, shared, collaborative or collective leadership. The evidence that this type of team and organisational leadership impacts positively on health outcomes, the patient experience and staff morale is growing (West et al., 2015). The strong focus on quality improvement and patient safety philosophies drawn largely from non-healthcare safetycritical industries (such as aviation and nuclear power) underpins health professionals' leadership and teamwork, all of which aligns with a change in culture that promotes patient-centredness and high quality, safe, compassionate care (Francis, 2013). A key challenge that remains for leaders is to facilitate, support and empower individuals and groups to speak out and act when they see poor or unsafe healthcare.

\section{TEACHING AND ASSESSING TEAMWORK}

Professional standards, frameworks and guidance exist to help practising doctors, educators and learners to work out what knowledge, skills and behaviours are required to work in and lead teams effectively. In 2012, the UK General Medical Council (GMC) published "Leadership and management for all doctors" (GMC, 2012) which was updated in 2013 around a set of standards expected of doctors (General Medical Council, 2013). These documents make it clear that effective teamworking and leadership is a professional obligation, expected of all doctors. In the UK, the Healthcare Leadership Model has been introduced which aims to help professionalise leadership at all levels of healthcare through defining expected leadership behaviours (NHS Leadership Academy, 2013). Such frameworks and standards help educators and learners to define what is expected from them. The Faculty of Medical Leadership and Management (FMLM) was established in 2011 to "promote the advancement of medical leadership, management and quality improvement at all stages of the medical career" (Faculty of Medical Leadership and Management, 2014). Membership provides access to events, expertise, coaching and mentoring support and a range of resources on leadership and management. In 2015, the FMLM Leadership and Management Standards for Medical Professionals were launched, again to assist medical leaders and managers to benchmark themselves against best practice. 
Worldwide, there has been an increase in defined leadership curricula and the provision of training in non-technical skills, professionalism, teamwork and leadership at all levels (O'Sullivan and McKimm, 2011). Teamwork and leadership have most commonly been subsumed under the banner of professionalism or non-technical skills in both undergraduate and postgraduate training. A number of teaching, learning and assessment methods have been developed to assist with these challenging educational aspects and evidence is emerging as to their effectiveness. In classroom based situations, facilitating learners to work in multiple teams with directed reflection and to engage in team building exercises can be beneficial, even before they are working clinically. Structured observation of clinical and other teams can also help provide insight into how teams work in practice, supported by presentations around teamworking, who works in teams and by consideration of patient safety issues when teams go wrong. For example, the Objective Structured Teaching Exercise (OSTE) has been developed to help faculty teach professionalism in clinical settings (Lu et al., 2014). Acquiring, practising and obtaining feedback on teamworking and leadership skills in a longitudinal developmental way is best undertaken through workplace based learning and assessment, including multi-source feedback, e.g. the Team Assessment of Behaviour (TAB) assessment in the Foundation Programme. Structured portfolios, which combine practical assessment with reflection can help support long term teamworking and leadership development.

Written tests include prioritisation tests and SJTs (Situational Judgement Tests) and whilst these can be helpful to provide a point in time assessment, unless they form part of a programmatic assessment, they do not aid long term practice development. Simulation provides many opportunities for practising skills and obtaining feedback on teamworking: through simple role play to engagement in high fidelity scenarios. For example, the University of Dundee has developed a postgraduate ward simulation exercise which assesses teamwork and leadership skills such as the "ability to prioritise competing demands, make safe informed decisions, prescribe safely and manage the care of three patients" (Stirling et al., 2012).

Khan et al. (2011) argue that the realistic simulated environment improves memory recall and application of this information. This supports the use of simulation-based assessment in terms of its catalytic effect on promoting positive behavioural change (Norcini et al., 2011). In addition, the simulated environment may provide the opportunity for learning in action, which appears to be a more effective learning method (O'Sullivan et al., 2012). In their study with medical undergraduates, Paskins and Peile (2010) found that students thought the use of mannequin-based simulation allowed them to develop teamwork skills not only as a more efficient team member but also as a leader. This finding supports the use of simulation to assess teamwork and leadership. The authors also found that students exposed to simulation were more confident in their clinical attachments and that they valued both repeated exposure and the feedback on their performance. Khan et al. (2011) 
also argue for the use of simulation in the longitudinal assessment of performance, helping to "to bridge the gap between the classrooms and the clinical environments".

\section{CONCLUSION}

Being able to work effectively in and lead teams as required is a vital skill for any health professional because effective teams form the cornerstone of high quality healthcare and contribute to health improvement and patient safety. Much research evidence tells us that high performing teams have clear shared goals, clarity of leadership that is authentic and distributed throughout the team, creative (not destructive) conflict and effective communication. Team leaders and members treat one another with respect and mutual trust, they know their strengths and roles and power, authority and control are not allowed to become issues. Team members are empowered to challenge if they feel patient safety or care is at risk. The challenge for leaders is to create and maintain this culture across and between multiple teams in what are often very complex and rapidly changing contexts.

\section{KEY POINTS}

- The ability to work within and between teams is a core leadership skill;

- Effective multidisciplinary teamwork contributes to improved health outcomes and a higher quality of care;

- Effective teams need clear goals, shared leadership and ongoing review of performance;

- Leaders need to be aware of power-distance, authority and control mechanisms which can undermine effective teamworking;

- Many teams operate dysfunctionally - 'pseudoteams' have few of the characteristics of successful teams, i.e. interdependency, reflexivity and shared objectives. 


\section{REFERENCES}

Anning, A, Cottrell, D, Frost, N, \& Green, J (2010) Developing multiprofessional teamwork for integrated children's services. Open University Press/McGraw-Hill International, Maidenhead.

Barrow, M, McKimm, J, Gasquoine, S, Rowe, D (2014) Collaborating in healthcare delivery: exploring conceptual differences at the 'bedside'. Journal of Interprofessional Care, early online 1-6, DOI: 10.3109/13561820.2014.955911

Chakraborti, C, Boonyasai, RT, Wright, SM, Kern, DE (2008) A systematic review of teamwork training interventions in medical student and resident education. Journal of General Internal Medicine, 23, 846-853.

Clinical Human Factors Group (2008) Just a Routine Operation teaching video. Available at http://chfg.org/articles-films-guides/films/just-a-routine-operation-teaching-video (accessed 4 February 2015).

Cooper, GS, Mclure, J (2005) Why Mothers Die 2000-2002: Executive Summary and Key Findings. Royal College of Obstetricians and Gynaecologists, London.

Ezziane, Z, Maruthappu, M, Gawn, L, Thompson, EA, Athanasiou, T, Warren, OJ (2012) Building effective clinical teams in healthcare, Journal of Health Organization and Management, 26 (4), $428-436$ http://dx.doi.org/10.1108/14777261211251508

Faculty of Medical Leadership and Management (2014) About Us: Faculty of Medical Leadership and Management. Available at www.fmlm.ac.uk/about-us (accessed 10 February 2015).

Francis, R (2013) Report of the Mid Staffordshire NHS Foundation Trust Public Inquiry. The Stationery Office, London.

General Medical Council (2012) Leadership and management for all doctors. General Medical Council, London. Available at www.gmc-

uk.org/guidance/ethical_guidance/management_for_doctors.asp (accessed 10 March 2015).

General Medical Council (2013). Good Medical Practice. General Medical Council, London. Available at www.gmc-uk.org/guidance/good_medical_practice.asp (accessed 10 March 2015).

Harmer, M. (2007) Independent Review on the care given to Mrs Elaine Bromiley on 29 March 2005. Clinical Human Factors Group. Available at 
www.chfg.org/resources/07_qrto4/Anonymous_Report_Verdict_and_Corrected_Timeline_ Oct_07.pdf (accessed 4 February 2015).

Hjortdahl, M, Ringen, A, Naess, AC, Wisborg, T (2009) Leadership is the essential nontechnical skill in the trauma team - results of a qualitative study. Scandinavian Journal of Trauma, Resuscitation and Emergency Medicine, 17, 48

Hofstede, G, Hofstede, GJ, Minkov, M (2010) Cultures and Organizations: Software of the Mind, 3rd ed.. McGraw-Hill, New York.

Institute for Healthcare Improvement (2015) How to improve: forming the team, available at http://www.ihi.org/resources/Pages/Howtolmprove/ScienceofImprovementFormingtheTeam.a spx (accessed 22 February 2015).

Keogh, B (2013) Review into the quality of care and treatment provided by 14 hospital trusts in England: Overview report. The Stationery Office, London.

Khan, K, Pattison, T, Sherwood, M (2011) Simulation in medical education. Medical Teacher, 33, 1-3.

Lu, WH, Myolona, E, Lane, S, Wertheim, WA, Baldelli, P, Williams, PC (2014) Faculty development on professionalism and medical ethics: The design, development and implementation of Objective Structured Teaching Exercises (OSTEs). Medical Teacher, 36, 876-882.

Mathur, N, Skelcher, C (2007) Evaluating democratic performance: methodologies for assessing the relationship between network governance and citizens. Public Administration Review, 67(2), 228-237.

Moneypenny M.J, Guha A, Mercer S.J, O'Sullivan H, McKimm J (2013) Don't follow your leader: challenging erroneous decisions. British Journal of Hospital Medicine. Vol 74, 12, 687690.

National Advisory Group on the Safety of Patients in England (2013). A promise to learn: a commitment to act. Improving the safety of patients in England (the Berwick report). available at

www.gov.uk/government/uploads/system/uploads/attachment data/file/226703/Berwick Report.pdf (accessed 10 March 2015).

Neily, J, Mills, PD, Young-Xu, Y, Carney, BT, West, P, Berger, DH, Mazzia, LM, Paull, DE, Bagian, JP (2010) Association between implementation of a medical team training program and surgical mortality. Journal of the American Medical Association, 304, 1693-1700. 
NHS Leadership Academy (2013) Healthcare Leadership Model available at www.leadershipacademy.nhs.uk/resources/healthcare-leadership-model/ (accessed 10 March 2015).

Norcini, JJ, Anderson, B, Bollela, V, Burch, V, Costa, MJ, Duvivier, R, Galbraith, R, Hays, R, Kent, A, Perrott, V, Roberts, TE (2011) Criteria for good assessment: Consensus statement and recommendations from the Ottawa 2010 Conference. Medical Teacher, 33, 206-214.

O'Leary, M.B., Mortensen, M. and Woolley, A.W. (2011) Multiple team membership: a theoretical model of its effects on productivity and learning for individuals and teams, Academy of Management Review, 36 (3), 461-478.

O'Sullivan, H, McKimm, J (2011) Medical leadership: an international perspective. British Journal of Hospital Medicine, 72, 638-641.

O'Sullivan, H, Van Mook, W, Fewtrell, R, Wass, V (2012) Integrating professionalism into the curriculum. Medical Teacher, 34, 155-157.

Paskins, Z, Peile, E (2010) Final year medical students' views on simulation-based teaching: a comparison with the Best Evidence Medical Education Systematic Review. Medical Teacher, 32, 569-577.

Porter-O'Grady, T. (2010), "Leadership for innovation: from knowledge creation to transforming health care", 1-31, in Porter-O'Grady, T, Malloch, K (Eds), Innovation Leadership: Creating the Landscape of Health Care, Jones and Bartlett, Boston, MA.

Quality AfHRa (2007) teamSTEPPS ${ }^{\mathrm{TM}}$ strategies and tools to enhance performance and patient safety. Rockville, MD.

Schyve, PM (2005) The changing nature of professional competence. Joint Commission Journal on Quality and Patient Safety. 31,185-202.

St Pierre, M, Scholler, A, Strembski, D, Brever, G (2012) Do residents and nurses communicate safety relevant concerns? Simulation study on the influence of the authority gradient. Der Anaesthesist, 61, 857-866.

Stirling, K, Hog, G, Ker, J, Anderson, F, Hanslip, J, Byrne, D (2012) Using simulation to support doctors in difficulty. The clinical teacher, 9, 285-289.

West, M, Armit, K, Loewenthal, L, Eckert, R, West, T, Lee, A (2015) Leadership and leadership development in healthcare: the evidence base. London, Faculty of Medical Leadership and Management.

West, MA, Lyubovnikova, J (2013) Illusions of Team Working in Health Care. Journal of Health Organization and Management, 27(1), 134-142. 
World Health Organisation (WHO) (2011) WHO Patient Safety Curriculum Guide, available at http://www.who.int/patientsafety/education/curriculum/en/ (accessed 22 February 2015).

Xyrichis A, Ream E (2008) Teamwork: a concept analysis. J Adv Nurs, 61:232-241. 